\title{
Helicobacter pylori: prospettive per un vaccino
}

\author{
Giuseppe Del Giudice, Rino Rappuoli \\ Centro Ricerche, Chiron Srl ,Via Fiorentina I, 53100 Siena
}

\section{SUMMARY}

Helicobacter pylori causes one of the most widespread infections worldwide: it affects more than $50 \%$ of the human population, and is responsible for serious gastric pathologies such as chronic gastritis, peptic ulcer, atrophic gastritis and, in some individuals, gastric cancer. Current treatments with antibiotics are efficacious, but encounters several drawbacks at the level of compliance, side effects, antibiotic resistance, etc. The availability of vaccines could contribute in reducing the burden of $H$. pylori associated diseases. Several bacterial antigens have been identified as virulence factors and proposed as potential vaccine candidates. Some of these antigens have been tested in experimental animal models of challenge with $H$. pylori. The experiments in animals have shown that prophylactic and therapeutic vaccination against $H$. pylori is indeed feasible. Several open questions still remain concerning the understanding of the host-microbe relationship and the quality of the immune response which should be induced in order to confer protective immunity in man. The answers to these questions will be crucial in helping the preparation of appropriate vaccine formulations able to efficaciously protect humans both prophylactically and therapeutically. A few clinical trials have been carried out so far with still limited results. Other trials in humans are in progress and are planned for the next few years. The final hope is that these new vaccines will show the expected efficacy against $H$. pylori and will permit the elimination of this pathogen which has cohabited with humans for more than 100,000 years.

\section{RELAZIONE}

\section{Il Batterio e l'infezione.}

L'Helicobacter pylori è un batterio micro-aerofilo, a forma di spirale e Gram-negativo. Inizialmente descritto nel 1982, appartiene alla lunga lista dei nuovi patogeni umani che sono stati scoperti nel corso degli ultimi 25 anni. L'Helicobacter pylori è un patogeno molto specializzato nel colonizzare esclusivamente la mucosa gastrica umana, un ambiente avverso in cui altri patogeni non sarebbero capaci di sopravvivere localizzandosi nel comparto extracellulare al di sotto dello strato mucoso.

Benché scoperto solo recentemente, l'evidenza genetica dimostra che l'Helicobacter pylori è un antico batterio che si è evoluto con l'uomo (1). L'infezione avviene in età precoce, molto probabilmente per via oro-fecale e/o per via oro-orale. La famiglia sembra essere l'ambiente in cui più frequentemente avviene la trasmissione dell'infezione (2).

Una volta stabilitosi, l'infezione persiste cronicamente, a meno che non venga effettuato un trattamento antibiotico. L'assenza di competizione nell'ambiente gastrico e l'abilità di stabilire una lunga colonizzazione fa dell'Helicobacter pylori un microrganismo vincente .

Infatti più del $50 \%$ della popolazione mondiale è affetta da Helicobacter Pylori e più del $90 \%$ nei paesi sviluppati (3). La maggior parte degli individui infettati da Helicobacter pylori non presentano sintomi. Nel 15\%-20\% dei casi di individui infettati, l'infezione cronica si evolve in ulcera peptica, in altri in gastrite atrofica, adenocarcino- ma gastrico, linfoma del tessuto linfoide associato alla mucosa gastrica ( MALT) (4).

Il trattamento dell'infezione da Helicobacter pylori viene effettuato solo nei pazienti sintomatici e consiste nella somministrazione giornaliera di un inibitore della pompa protonica e di due antibiotici per una o due settimane (5). Malgrado la buona efficacia ( $80 \%$ o più) il trattamento farmacologico si imbatte in diversi ordini di problemi. L'alto numero di compresse somministrate giornalmente può causare seri problemi al paziente. Anche gli effetti collaterali non sono rari. Nel mondo è stato riscontrato un numero sempre crescente di ceppi resistenti agli antibiotici.

In alcune aree di alta trasmissione sono stati riscontrati anche molti casi di reinfezione.

Visto che i trattamenti vengono somministrati ai pazienti con sintomi, gli individui senza sintomi sono ancora sottoposti al rischio di subire importanti complicazioni quali la gastrite atrofica ed il cancro gastrico. Tutti questi problemi potrebbero essere superati dalla disponibilità di vaccini profilattici e/o vaccini terapeutici contro l'Helicobacter pylori, già allo studio da parte di diversi gruppi, seguendo differenti approcci.

La conoscenza dell'intero genoma batterico potrebbe essere particolarmente utile nell'identificazione di potenti vaccini (6), benché i candidati sino ad ora testati siano stati identificati prima dell'era genomica. Recenti analisi farma-economiche hanno mostrato con chiarezza quanto potrebbe essere economicamente vantaggioso l'uso di vaccini profilattici contro l'Helicobacter pylori nella prevenzione di gravi complicazioni 
dell' infezione come l'ulcera peptica ed il cancro gastrico $(7,8)$.

\section{L'importanza di alcuni antigeni in virulenza e patogenesi}

Molto spesso gli antigeni vengono selezionati come potenziali vaccini grazie ad alcune loro peculiari caratteristiche. Gli antigeni candidati per un vaccino, ad esempio, sono esposti in superficie quindi facilmente attaccabili dal sistema immune e sono abbondantemente espressi risultando più facilmente immunogenici.

Essi sono ben conservati in tutti gli isolati batterici per indurre una risposta immune protettiva ad ampio spettro e rappresentano fattori di virulenza coinvolti nella patogenesi dell' infezione in modo tale che la loro attivazione possa bloccare la colonizzazione e/o la sopravvivenza dei batteri.

Nel caso dell' Helicobacter pylori, un potenziale vaccino che apparentemente soddisfa tutte queste caratteristiche è l'ureasi (9). Questo enzima è ben conservato e consiste in due subunità.

UreA di $27 \mathrm{kDa}$ e UreB di $62 \mathrm{kDa}$. L'ureasi che è liberata dal batterio attraverso l'autolisi è molto abbondante e rappresenta il 5\% $-10 \%$ del totale del contenuto proteico. L'Ureasi è coinvolta nella colonizzazione batterica: i ceppi isogenici ureasi negativi sono incapaci di colonizzare lo stomaco dei maialini gnotobiotici. Sembra essere coinvolta anche nell'attivazione e adesione delle cellule infiammatorie nel sito delle lesioni gastriche. Malgrado l'abbondanza l'ureasi non sembra essere particolarmente immunogenica durante l'infezione naturale. Gli anticorpi anti-ureasi vengono ritrovati approssimativamente nel 50\% degli individui infettati con Helicobacter pylori (10), e pochissime cellule T CD4+ specifiche per l' ureasi vengono usualmente isolate dalla mucosa gastrica di individui con gastrite cronica o ulcera peptica $(11,12)$. Sino ad ora l'ureasi è stato l'antigene più studiato come potenziale vaccino contro l'Helicobacter pylori negli animali ed è stato anche utilizzato in sperimentazioni cliniche nell'uomo ( vedi sotto). Un altro antigene ben conservato è la proteina attivatrice dei neutrofili (NAP). Questa proteina di $17 \mathrm{kDa}$, la cui struttura presenta $4 \alpha$-eliche, si riunisce per formare dodecameri. NAP ha la capacità di legare il ferro, indurre chemiotassi, attivare neutrofili e monociti e stimolare la produzione di intermedi attivi dell' ossigeno. Questa attività è potenziata da IFN- $\gamma$ e TNF $\alpha$ (13). Così' NAP può indurre una infiammazione locale gastrica attraverso il reclutamento locale e l'attivazione di neutrofili e monociti. Gli isolati di Helicobacter pylori sono stati suddivisi in due grandi categorie. I cosiddetti ceppi di tipo I sono associati con le più gravi complicazioni dell' infezione ( ulcera peptica, gastrite atrofica, cancro gastrico), mentre i ceppi del tipo II vengono più frequentemente isolati negli individui con leggere forme di gastrite. I ceppi del tipo I contengono un gene ( $\operatorname{cagA}$ ) che codifica il cosiddetto gene associato alla citotossina ( cagA) che risiede in un frammento di $40 \mathrm{~kb}$ presente nel tipo I, ma non nel tipo II (1). Questa regione ( cag ) e un' isola patogenica (PAI) simile a quella trovata in altri patogeni, come Escherichia coli enteropatogenica, Bordetella pertussis etc. La maggior parte (70\%) di isolati clinici sono CagA positivi. CagA è altamente immunogenica sia a livello anticorpale che cellulare; questa risposta immune è stata considerata come un segno di infezione grave. La cag PAI dell' Helicobacter pylori induce la produzione di IL-8 da parte delle cellule epiteliali, l'attivazione di NF-Kb, il rimodellamento del citoscheletro , la formazione di protrusioni cellulari etc. La cosa importante è che cag PAI media la traslocazione di CagA in cellule eucariotiche, dove CagA viene fosforilato $(14,15)$. Questo può spiegare l'associazione che è stata scoperta fra l' infezione da ceppi CagA positivi e lo sviluppo di importanti patologie gastriche. In accordo con osservazioni epidemiologiche fatte in umani infettati ed in gerbilli mongoli è stato visto che i ceppi CagA positivi causano importanti patologie gastriche, incluso il cancro, mentre i ceppi CagA negativi causano solo gastriti leggere (16). L'inoculazione attiva di CagA dentro la cellula eucariotica rappresenta un nuovo meccanismo di interazione fra un patogeno extracellulare stretto e la cellula e può anche spiegare l'alta immunogenicità di CagA riscontrata in individui infettati. L'Helicobacter Pylori produce una citotossina che induce la vacuolazione di cellule in vitro (tossina vacuolizzante VacA). La proteina matura contiene una porzione di $37 \mathrm{kDa}$ essenziale per l'attività tossica, e una di $58 \mathrm{kDa}$ che lega la cellula bersaglio $(15,17)$. I monomeri VacA oligomerizzano a formare strutture esa e eptameriche a forma di fiore.

La sua sequenza è ben conservata fra differenti isolati, ad eccezione delle regioni mediane della parte di $58 \mathrm{kDa}$, che esprime variazioni alleliche . Dati clinici e sperimentali mostrano chiaramente come entrambe gli alleli sono tossici (17); potrebbero ben rappresentare l'espressione evolutiva a livello batterico di un polimorfismo genetico a livello del recettore specifico di questa tossina nella cellula ospite.

\section{Un'insufficiente risposta naturale all'Helicobacter pylori}

Le nostre conoscenze sull'infezione dell'Helicobacter pylori derivano soprattutto dai pazienti adulti infettati cronicamente da molto 
tempo. Molto poco conosciamo sui primi stadi dell'infezione durante l'età pediatrica, così come della storia naturale, dallo stabilirsi di una colonizzazione all'insorgere evidente di malattie gastriche. L'Infezione da Helicobacter pylori induce una risposta anticorpale (rivelabile nel siero e a livello mucosale) che viene utilizzata per scopi diagnostici. Comunque la risposta immune naturale indotta dall'infezione non sembra conferire immunità protettiva. Ad esempio, $\mathrm{i}$ furetti curati con successo da un' infezione naturale da H.mustelae sono ancora suscettibili alla reinfezione con lo stesso batterio (18). Alte percentuali di reinfezione sono state riscontrate in individui che vivono in aree di alta prevalenza di infezione da Helicobacter pylori (19). Al contrario sussiste l'evidenza che le lesioni gastriche indotte da Helicobacter pylori potrebbero essere mediate dalla risposta immune contro il batterio (20). In ogni caso, gli eventi che conducono all'instaurarsi di una risposta immune all'Helicobacter pylori ed allo sviluppo di lesioni gastriche, sono ancora molto poco conosciuti. L'infezione naturale da Helicobacter pylori comporta una regolazione positiva della molecola costimolatoria B7-2 e dell'espressione di MHC di classe II sulle cellule epiteliali, con un incremento in numero di cellule mononucleate attivate che infiltrano la lamina propria. In aggiunta c'è la comparsa di linfociti T CD4 la maggior parte con un fenotipo di cellule della memoria attivate e di linfociti B. Queste cellule iniziano ad organizzarsi a formare dei follicoli linfoidi che, ad uno stadio più avanzato, diventano macroscopicamente evidenti e rappresentano il sito dove il linfoma gastrico può eventualmente svilupparsi in alcuni individui.

Alcune linee di evidenza hanno mostrato chiaramente che le cellule $\mathrm{T}$ gastriche $\mathrm{CD} 4+$, provenienti da animali infettati sperimentalmente e da individui infettati naturalmente, esibiscono un fenotipo funzionale di tipo Th1, con una produzione di citochine come IFN- $\gamma$,TNF- $\alpha$,TGF- $\beta$,IL$18(21,22,23)$. Cloni di cellule T Th1 CD4 sono molto più frequentemente isolati dalla mucosa gastrica dei pazienti affetti da ulcera peptica, mentre i cloni Th0/Th2 vengono ritrovati più frequentemente in pazienti affetti da una leggera forma di gastrite $(11,12)$. Cosa molto importante è che questi cloni erano frequentemente specifici per CagA, e pochissimi riconoscevano ureasi e altri antigeni. Un'attivazione predominante di cellule T Th1 CD4 è stato anche osservata in infezioni da Helicobacter pylori in modelli murini $(24,25)$.

L'ipotesi che gli eventi patologici osservati nello stomaco possano essere mediati dalla risposta immune scatenata dall'Helicobacter pylori è provata dagli studi condotti in ceppi di topi knockout. Infatti la patologia gastrica è più importante in topi knock-out per IL-10 infettati con $\mathrm{H}$. felis (26) e in topi knock-out per IL-4 (27) o IRF-1 (28) infettati con Helicobacter pylori, suggerendo un ruolo attivo di citochine Th1 nell'induzione della patologia gastrica e/o anche che citochine di tipo Th2 possano neutralizzare gli effetti proinfiammatori delle citochine di tipo Thl.

I topi infettati naturalmente con il nematode intestinale Heligmosomoides polygyrus, perciò naturalmente incline ad una risposta immune polarizzata verso il fenotipo funzionale di tipo Th2, quando sono sperimentalmente infettati con $H$. felis, esibiscono una patologia gastrica significativamente meno grave di quella osservata in topi non co-infettati con un parassita intestinale (29). Possiamo concludere quindi che l'Helicobacter pylori induce una patologia gastrica associata con una forte risposta immune di tipo Th1.

In aggiunta, diversi antigeni di $H$. pylori (esempio NAP, cag PAI, ureasi etc) possono attivamente intervenire nel potenziare la spirale degli eventi pro-infiammatori mediati da IFN- $\gamma$ ed altre citochine e chemiochine, inclusa IL-8. Altri antigeni, come NAP, possono direttamente (o sinergicamente con IFN- $\gamma$ e TFN- $\alpha$ ) aumentare il reclutamento e l'attivazione delle cellule infiammatorie nel sito della colonizzazione.

Infine va detto che alcuni epitopi batterici, ad esempio dell' LPS, sono condivisi con quelli propri dell'ospite come quelli dei gruppi sanguigni di Lewis che sono espressi dalla maggior parte delle cellule, incluse le cellule epiteliali gastriche. Anticorpi contro LPS di $H$. pylori capaci di reazione incrociata contro tessuti gastrici sono stati dimostrati in pazienti naturalmente infettati ed in animali sperimentalmente infettati (30). La rilevanza di questi anticorpi cross-reattivi nell'induzione di gastriti croniche a seguito dell'infezione da H.pylori rimane comunque ancora da provare. 4. La fattibilità di vaccinazioni profilattiche $e$ terapeutiche in modelli animali.

Esiste un gran numero di prove che una protezione contro l'infezione da Helicobacter pylori possa essere raggiunta in modelli animali sia dal punto di vista profilattico che terapeutico. Questo è stato dimostrato in topi infetti da H.felis ( una specie che non infetta l'uomo e non esprime fattori di virulenza come VacA e cag PAI) immunizzati oralmente con lisati batterici insieme alla tossina del colera (CT) come adiuvante mucosa. Questo è stato poi dimostrato con ceppi di H. pylori isolati di fresco in pazienti affetti da ulcera peptica ed adattati al topo. La protezione profilattica e terapeutica contro l'Helicobacter pylori è stata dimo- 
strata con diversi antigeni nativi o ricombinanti come l'Ureasi (e subunità ricombinanti), proteine dello shock termico VacA, CagA, NAP, catalase ed altre native e ricombinanti (31). Altri antigeni protettivi potranno essere identificati grazie alle informazioni derivate dal genoma dell' Helicobacter pylori. La maggior parte degli studi di vaccinazione condotti sino ad ora si sono focalizzati sull' immunizzazione via orale. Studi su modelli animali, comunque, dimostrano che altre vie di immunizzazione (per esempio intranasale, rettale o parenterale) possano ugualmente essere efficaci nel conferire protezione contro l'Helicobacter pylori o H. felis (31). Comunque, risultati contraddittori in merito alla protezione sono stati ottenuti in scimmie immunizzate con ureasi. Se la protezione profilattica e quella terapeutica contro l'Helicobacter pylori è stata realizzata in diversi modelli animali usando diversi antigeni e diverse strade di immunizzazione, il meccanismo immune che media questa protezione rimane ancora un enigma. Inizialmente è stata ipotizzata la presenza di anticorpi specifici ( $\operatorname{IgA}$ o $\mathrm{IgG}$ ) prodotti localmente nell'ambiente gastrico. Comunque la protezione contro l'Helicobacter pylori può essere anche raggiunta in topi incapaci di produrre IgA. Possiamo inoltre aggiungere che gli umani, in cui vi sia una deficienza congenita di IgA, non soffrono di una forma più grave di patologia gastrica da Helicobacter pylori (32).

Infine, la protezione contro l'Helicobacter pylori o $H$. felis può essere raggiunta in topi $\mu$-MT, totalmente incapaci di produrre anticorpi (33). La protezione richiede la presenza di un'attività di cellule CD4+ ristrette per classe II dell' MHC. L'esatta e funzione di queste cellule CD4 + rimane ancora poco chiara.

È stato proposto che cellule T CD4 +aventi il fenotipo $\alpha 4 \beta 7^{\text {hi }}$ siano richiesti per la protezione contro $H$.felis dopo l'immunizzazione orale .

E' stato mostrato che la localizzazione di queste cellule nella mucosa gastrica era mediato dall'adiuvante mucosale usato, il $\mathrm{CT}$, ma non da $H$. felis; in aggiunta trattamenti in vivo con un anticorpo monoclonale contro integrina $\alpha 4 \beta 7$ sopprimono la protezione. È stato mostrato come una risposta polarizzata $\mathrm{CD} 4+\mathrm{Th} 2$ sia preferibilmente indotta dopo l'immunizzazione orale con antigeni di Helicobacter pylori e adiuvanti mucosali come CT (24), LT, o mutanti non tossici di LT (31) . In accordo con la constatazione che topi knock-out al livello di citochine di tipo Th2 come IL-4 e IL-10 hanno una grave patologia gastrica dopo l'infezione da Helicobacter pylori, $(26,27)$ può essere proposto che l'immunizzazione protettiva induce citochine di tipo Th2 che agiscono inibendo l'attivazione delle cellule Th1 e dei macrofaghi per la produzione di citochine proinfiammatorie che sono normalmente prodotte durante l'infezione da Helicobacter pylori. La conoscenza del meccanismo immunologico, che è alla base della protezione sia profilattica che terapeutica contro l' Helicobacter pylori, sarà di critica importanza per il disegno di vaccini efficaci da testare in sperimentazioni cliniche che avranno luogo nei prossimi anni.

\section{L'esperienza clinica con i vaccini contro l'Helicobacter pylori: presente e futuro. Sino ad ora pochi esperimenti sono stati condotti in esseri umani.}

Due studi nei quali è stata usata la Salmonella attenuata che esprime ureasi, hanno dimostrato poca o nulla immogenicità $(35,36)$. Uno studio è condotto con la somministrazione orale di ureasi insieme a LT wild-type come adiuvante in soggetti infettati da Helicobacter pylori e con gastrite cronica (37). Due terzi dei soggetti hanno sviluppato diarrea a causa di LT wild-type. IgA sieriche specifiche per l'ureasi e cellule secernenti anticorpi circolanti sono state trovate nei soggetti che avevano ricevuto le più alte dosi di antigeni (60 o $180 \mathrm{mg}$ dati 4 volte con cadenza settimana1e). Una parziale riduzione del numero delle colonie batteriche è stata osservata, paradossalmente, in un gruppo di soggetti che aveva ricevuto la più bassa dose di ureasi $(20 \mathrm{mg}$ ). Infine, l'immunizzazione non ha causato miglioramenti nell'infiammazione gastrica pre-esistente. Le ragioni dei risultati parziali di questi esperimenti non sono chiare. Potrebbero essere legate al regime di vaccinazione impiegato, agli antigeni usati, agli adiuvanti utilizzati e anche alle difficoltà inerenti il tipo di approccio terapeutico di vaccinazione, non ancora ben conosciuto. Più recentemente sono stati pubblicati i risultati di una sperimentazione in cui veniva usato un vaccino orale costituito da cellule intere inattivate e con un mutante LT come adiuvante (38). Il vaccino somministrato sia a soggetti infetti che a soggetti non infetti era immunogenico ma induceva diarrea (causa l'adiuvante impiegato) e non dimostrava efficacia terapeutica negli individui infetti. Attualmente, ed in un futuro non molto lontano, verranno testati negli uomini formulazioni di vaccini sia profilattici che terapeutici. Gli studi forniranno risposte ai quesiti più cruciali sull'interazione tra microbo e ospite. La speranza finale è che questi nuovi vaccini mostrino l'efficacia attesa contro le patologie causate dall'Helicobacter pylori e permettano l'eliminazione di questo patogeno che ha coabitato con l'uomo per più di 100.000 anni. 


\section{BIBLIOGRAFIA}

1. Covacci, A., Telford, J.L., Del Giudice, G., Parsonnet, J., and Rappuoli, R. (1999) Helicobacter pylori virulence and genetic geography. Science 284, 13281333.

2. Tindberg, Y., Bengtsson, C., Granath, F., Blennow, M., Nyrén, O., and Grandström, M. (2001) Helicobacter pylori infection in Swedish school children: lack of evidence of child-to-child transmission outside the family. Gastroenterology 121, 310-316.

3. Parsonnet, J. (1995) The incidence of Helicobacter pylori infection. Aliment. Pharmacol. Ther. 9 (suppl. 2), 45-51.

4. Uemura, N., Okamoto, S., Yamamoto, S., Matsumura, N., Yamaguchi, S., Yamakido, M., Taniyama, K., Sasaki, N., and Schlemper, R.J. (2001) Helicobacter pylori infection and the development of gastric cancer. N. Engl. J. Med. 345, 784-789.

5. Graham, D.Y. (2000) Therapy of Helicobacter pylori: current status and issues. Gastroenterology 118, S2-S8.

6. Alm, R.A., and Trust, T.J. (1999) Analysis of the genetic diversity of Helicobacter pylori: the tale of two genomes. J. Mol. Biol. 77, 834-846.

7. Rupnow, M.F.T., Owens, D.K., Shachter, R., and Parsonnet, J. (1999) Helicobacter pylori vaccine development and use: a cost-effectiveness analysis using the Institute of Medicine methodology. Helicobacter 4, 272-280.

8. Stratton, K.R., Durch, J.S., and Lawrence, R.S., eds. (2000) Vaccines for the 21st Century. A tool for decisionmaking. National Academy Press, Washington DC.

9. Mobley, H.L.T. (2001) Urease. In: Helicobacter pylori. Physiology and genetics (H.L.T. Mobley, G.L. Mendz, and S.L. Hazell, eds.), pp. 179-191. ASM Press, Washington DC.Leal-Herrera, Y., Torres, J., Perez-Perez, G., Gomez, A., Monath, T., TapiaConyer, R., and Munoz, O. (1999) Serologic IgG response to urease in Helicobacter pylori-infected persons from Mexico. Am. J. Trop. Med. Hyg. 60, 587-592.

10. D’Elios, M.M., Manghetti, M., De Carli, M., Costa, F., Baldari, C.T., Burroni, D., Telford, J.L., Romagnani, S., and Del Prete, G. (1997) T helper 1 effector cells specific for Helicobacter pylori in the gastric antrum of patients with peptic ulcer disease. J. Immunol. 158, 962-967.

11. D'Elios, M.M., Manghetti, M., Almerigogna, F., Amedei, A., Costa, F., Burroni, D., Baldari, C.T., Romagnani, S., Telford, J.L., and Del Prete, G. (1997) Different cytokine profile and antigen-specificity repertoire in Helicobacter pylori-specific T cell clones from the antrum of chronic gastritis patients with or without peptic ulcer. Eur. J. Immunol. 27, 17511755.

12. Satin, B., Del Giudice, G., Della Bianca, V., Dusi, S., Laudanna, C., Tonello, F., Kelleher, D., Rappuoli, R., Montecucco, C., and Rossi, F. (2000) The neutrophilactivating protein (HP-NAP) of Helicobacter pylori is a protective antigen and a major virulence factor. J. Exp. Med. 191, 1567-1476.

13. Covacci, A., and Rappuoli, R. (2000) Tyrosine-phosphorylated bacterial proteins: Trojan horses for the host cells. J. Exp. Med. 191, 587-592.

14. Montecucco, C., and Rappuoli, R. (2001) Living dangerously: how Helicobacter pylori survives in the human stomach. Nature Rev. Mol. Cell Biol. 2, 457466.

15. Ogura, K., Maeda, S., Nakao, M., Watanabe, T., Tada,
M., Kyutoku, T., Yoshida, H., Shiratori, Y., and Omata, M. (2000) Virulence factors of Helicobacter pylori responsible for gastric diseases in Mongolian gerbils. J. Exp. Med. 192, 1601-1610.

16. Reyrat, J.M., Pelicic, V., Papini, E., Montecucco, C., Rappuoli, R., and Telford, J.L. (1999) Towards deciphering the Helicobacter pylori cytotoxin. Mol. Microbiol. 34, 197-204.

17. Batchelder, M., Fox, J., Hayward, A., Yan, L., Shames, B., Murphy, J.C., and Palley, L. (1996) Natural and experimental Helicobacter mustelae reinfection following successful antimicrobial eradication in ferrets. Helicobacter 1, 34-42.

18. Ramirez-Ramos, A., Gilman, R.H., Leon-Barua, R., Recavarren-Arce, S., Watanabe, J., Salazar, G., Checkley, W., McDonald, J., Valdez, Y., Cordero, L., and Carrazco, J. (1997) Rapid recurrence of Helicobacter pylori infection in Peruvian patients after successful eradication. Gastrointestinal Physiology Working Group of the Universidad Peruana Cayetano Heredia and the Johns Hopkins University. Clin. Infect. Dis. 25, 1027-1031.

19. Ernst, P.B., and Gold, B.D. (2000) The disease spectrum of Helicobacter pylori: the immunopathogenesis of gastroduodenal ulcer and gastric cancer. Annu. Rev. Microbiol. 54, 615-640.

20. Bamford, K.B., Fan, X., Crowe, S.E., Leary, J.F., Gourley, W.K., Luthra, G.K., Brooks, E.G., Graham, D.Y., Reyes, V.E., and Ernst, P.B. (1998) Lymphocytes in the human gastric mucosa during Helicobacter pylori infection have a $\mathrm{T}$ helper cell 1 phenotype. Gastroenterology 114, 482-492.

21. Lindholm, C., Quiding-Jarbrink, M., Lonroth, H., Hamlet, A., and Svennerholm, A.M. (1998) Local cytokine response in Helicobacter pylori infected subjects. Infect. Immun. 66, 5964-5971.

22. Tomita, T., Jackson, A.M., Hida, N., Hayat, M., Dixon, M.F., Shimoyama, T., Axon, A.T.R., Robinson, P.A., and Crabtree, J.E. (2001) Expression of IL-18, a Th1 cytokine, in human gastric mucosa is increased in Helicobacter pylori infection. J. Infect. Dis. 183, 620-627.

23. Saldinger, P.F., Porta, N., Launois, P., Louis, J.A., Wanders, G.A., Bouzourene, H., Michetti, P., Blum, A.L., and Corthesy-Theulaz, I.E. (1998) Immunization of BALB/cmice with Helicobacter urease B induces a T helper 2 response absent in Helicobacter infection. Gastroenterology 115, 891-897.

24. Eaton, K.A., Mefford, M., and Thenenot, T. (2001) The role of T cell subsets and cytokinesin the pathogenesis of Helicobacter pylori gastritis in mice. J. Immunol. 166, 7456-7461.

25. Berg DJ, Lynch NA, Lynch RG, Mauricella DM: Rapid development of severe hyperplastic gastritis with gastric epithelial dedifferentiation in Helicobacter felis-infected IL-10-/- mice. Am. J. Pathol. (1998) 152:1377-1386.

26. Smythies, L.E., Waites, K.B., Lindsey, J.R., Harris, P.R., Ghiara, P., and Smith, P.D. (2000) Helicobacter pylori-induced mucosal inflammation is Th1 mediated and exacerbated in IL-4, but not IFN-g, gene-deficient mice. J. Immunol. 165, 1022-1029.

27. Sommer, F., Faller, G., Roellinghoff, M., Kirchner, T., Mak, T.W., and Lohoff, M. (2001) Lack of gastritis and of an adaptive immune response in interferon regulatory factor-1-deficient mice infected with Helicobacter pylori. Eur. J. Immunol. 31, 396-402.

28. Fox, J.G., Beck, P., Dangler, C.A., Whary, M.T., Wang, T.C., Shi, H.N., and Nagler-Anderson, C. 
(2000) Concurrent enteric helminth infection modulates inflammation and gastric immune responses and reduces helicobacter-induced gastric atrophy. Nature Med. 6, 536-542.

29. Moran, A.P., and Prendergast, M.M. (2001) Molecular mimicry in Campylobacter jejuni and Helicobacter pylori lipopolysaccharides: contribution of gastrointestinal infections to autoimmunity. J. Autoimmunity 16, 241-256.

30. Del Giudice, G., Covacci, A., Telford, J.L., Montecucco, C., Rappuoli, R.: The design of vaccines against Helicobacter pylori and their development. Annu. Rev. Immunol. (2001) 19:523-563.

31. Bogstedt, A.K., Nava, S., Wadstrom, T., and Hammarstrom, L. (1996) Helicobacter pylori infection in IgA deficiency: lack of role for the secretory immune system. Clin. Exp. Immunol. 105, 202-204.

32. Ermak, T.H., Giannasca, P.J., Nichols, R., Myers, G.A., Nedrud, J., Weltzin, R., Lee, C.K., Kleanthous, H., and Monath, T.P. (1998) Immunization of mice with urease vaccine affords protection against Helicobacter pylori infection in the absence of antibodies and is mediated by MHC class II-restricted responses. J. Exp. Med. 188, 2277-2288.

33. Michetti, M., Kelly, C.P., Kraehenbuhl, J.P., Bouzourene, H., and Michetti, P. (2000) Gastric mucosal ?4?7-integrin-positive CD4 T lymphocytes and immune protection against Helicobacter infection in mice. Gastroenterology 119, 109-118.

34. Di Petrillo, M.D., Tibbetts, T., Kleanthous, H., Killeen, K.P., and Hohmann, E.L. (1999) Safety and immunogenicity of $p h o P / p h o Q$-deleted Salmonella typhi expressing Helicobacter pylori urease in adult volunteers. Vaccine 18, 449-459.

35. Angelakopoulos, H., and Hohmann, E.L. (2000) Pilot study of phoP/phoQ-deleted Salmonella enterica serovar Typhimurium expressing Helicobacter pylori urease in adult volunteers. Infect. Immun. 68, 2135-2141.

36. Michetti, P., Kreiss, C., Kotloff, K., Porta, N., Blanco, J.L., Bachmann, D., Herranz, M., Saldinger, P.F., Corthesy-Theulaz, I., Losonsky, G., Nichols, R., Simon, J., Stolte, M., Ackerman, S., Monath, T.P., snd Blum, A.L. (1999) Oral immunization with urease and Escherichia coli heat-labile enterotoxin is safe and immunogenic in Helicobacter pylori-infected adults. Gastroenterology 116, 804-812.

37. Kotloff, K., Sztein, M.B., Wasserman, S.S., Losonsky, G., Di Lorenzo, S.C., and Walker, R.I. (2001) Safety and immunogenicity of oral inactivated whole-cell Helicobacter pylori vaccine with adjuvant among volunteers with or without subclinical infection. Infect. Immun. 69, 3581-3590. 